\title{
Catastrophic effects of climate change on children's health start before birth
}

\author{
Susan E. Pacheco
}

Department of Pediatrics, The University of Texas Health Science Center, Houston, Texas, USA.

W hen I first paid attention to the magnitude of the climate crisis in 2006, it was hard to accept that I had been indifferent to the problem for so many years. As a pediatrician, it did not take long to realize that children, whose bodies and minds are still developing and who are dependent on adults for care, are the most tragic casualties of the climate crisis. The shift in weather patterns, increased heat, heat waves, and drought; the resulting wildfires, increased storm intensity and flooding, crop failure and lower nutritional value, and shifting pattern of infectious vectors; and the resulting air pollution from continued use of fossil fuels impose a heavy burden in children, whose inherent physical and emotional immaturity makes them more vulnerable to these insults.

\section{Adverse effects on children}

The Intergovernmental Panel on Climate Change predicts that climate change will likely affect humans in multiple adverse ways: (a) increased mortality and morbidity associated with heat waves and fires and increased risks of food- and water-borne diseases (very high confidence levels), (b) increased risk of undernutrition due to diminished food production in poor regions and adverse effects of climate-altering pollutants besides $\mathrm{CO}_{2}$ (high confidence levels), and (c) increase in vector-borne diseases (medium confidence levels) (1). This translates to future generations of children who will continue to live in a deteriorating environment and the perpetuation of ongoing climate-related adverse health outcomes, such as air pollution-related increase in respiratory diseases and airway infections, heat-related illness, accidents, malnutrition, vector-transmitted diseases, psychological trauma, and mental health problems, among many others
(2). A child's vulnerability is magnified by dependence on adults who may have been adversely affected by a shared extreme weather event. After Hurricane Maria hit Puerto Rico in 2017, many adult members of family units experienced PTSD, depression, and anxiety after living for weeks and months without access to clean water, electricity, and in many cases, basic medical care. For this reason, some were not capable of meeting the physical and emotional demands that such a disaster imposed on their children.

Unmitigated, many more children will endure catastrophic conditions because of the climate crisis. Weather-related disasters, such as floods, droughts, hurricanes, and cyclones, directly affected an estimated 66.5 million children worldwide every year from 1990-2000, and such events are expected to affect 175 million children per year over the upcoming decades (3). The International Food Policy Research Institute projects that 25 million more children will be malnourished by 2050 . Ninety-three percent of all children in the world are exposed to levels of fine particulate matter (PM2.5) higher than the World Health Organization (WHO) air quality recommendations, the majority of them living in low- to medium-income countries. The inflicted damage is not trivial. For example, children living in megacities such as Mexico City, who have been exposed to air pollution all their lives, have evidence of systemic and neurological inflammation, early histological hallmarks for Alzheimer's and Parkinson's diseases, volumetric decreases in brain tissue, and cognitive dysfunction (4).

\section{Fetal programming and transgenerational inheritance The "fingerprint" of climate change on future generations of children begins before}

Conflict of interest: The author has declared that no conflict of interest exists.

Copyright: (c) 2020, American Society for Clinical Investigation.

Reference information: J Clin Invest. 2020;130(2):562-564. https://doi.org/10.1172/JCI135005.

they are born, as pregnant mothers endure environmental crises similar to those their children will experience after they are born. Maternal exposure to climaterelated disruptions, such as floods, droughts, famine, heat extremes, air pollution, and increased infection risk, as well as the stress associated with extreme weather events, population displacement, and other environmental crises, can shape the developing fetus any time during gestation. These outcomes, evident from birth to adulthood (Figure 1), may be mediated by epigenetic modulation, physiological resetting of hormonal feedback loops such as the hypothalamic-pituitary-adrenal axis, inflammation, oxidative stress, and altered development of somatic structures, among others. The increased risk of health problems associated with maternal exposures during pregnancy, such as preterm delivery or LBW; neurodevelopmental disorders, such as ASD, schizophrenia, and mood disorders; heart conditions; asthma; aero allergies and eczema; and metabolic conditions, type 2 diabetes, or weight problems, increases the morbidity and mortality that climate change will bring to children.

\section{Maternal stress, nutrition, and exposure to extreme weather events}

Maternal stress, caused by adverse life events or exposure to natural disasters, is associated with adverse pregnancy outcomes. In utero exposure to maternal stress due to bereavement and weather disasters, such as Hurricane Katrina, the 1998 Quebec ice storm, or the 2008 Iowa flood, have been associated with preterm birth, LBW, and metabolic derangements, such as elevated BMI and central adiposity, in children (5, 6). Neurodevelopmental conditions, such as ASD and ADHD, mood disorders, and schizophrenia, have been observed also. Gestational undernutrition has been associated with greater risk of pulmonary disease, stroke, and cardiovascular disease in adults. 


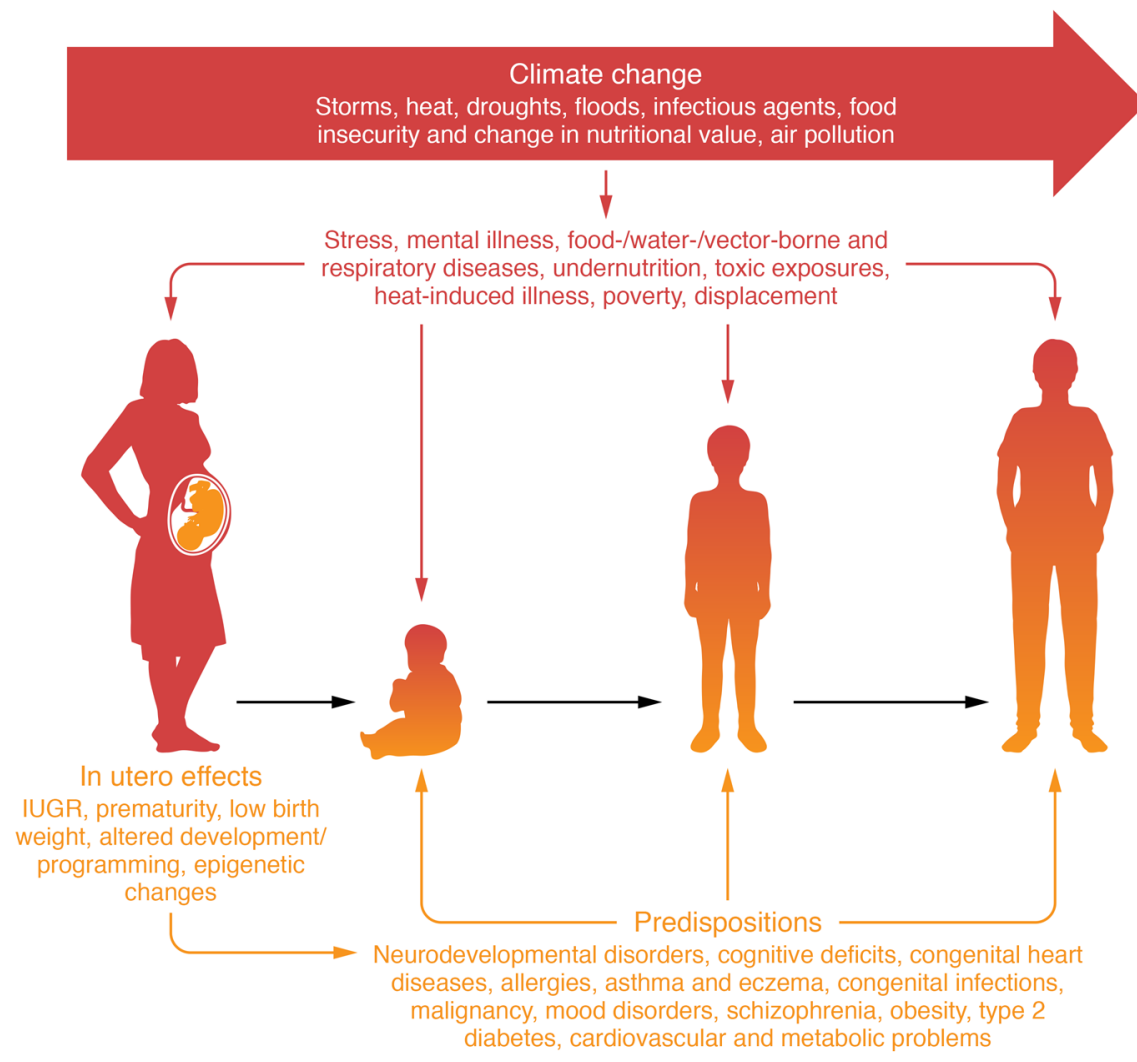

Figure 1. Adverse effects of climate change on children's health start before they are born.

Pregnant women exposed to climate change are affected by stress, respiratory disease, nutritional issues, infections, heat-induced illnesses, and poverty. Children are affected in utero, leading to increased risk of intrauterine growth retardation (IUCR), low birth weight (LBW), and prematurity. Fetal programming and epigenetic changes during development also cause long-term adverse effects induced by climate change as children grow due to increased predisposition to a number of conditions, such as attention deficit hyperactivity disorder (ADHD), autism spectrum disorder (ASD), and other neurodevelopmental disorders; cognitive deficits; congenital heart diseases; allergic rhinitis; asthma and eczema; congenital infections; malignancy; mood disorders; schizophrenia; obesity; type 2 diabetes; and cardiovascular and metabolic problems.

Likewise, children conceived during the 1944 Dutch famine had increased incidence of schizophrenia and depression as well as predisposition to coronary heart disease and type 2 diabetes as adults $(7,8)$. Based on these data, climate change is likely to impair the prospect of health and survival that every child deserves. Indeed, recent data suggest that maternal exposure to extreme heat in the first trimester is associated with increased risk for congenital heart disease (CHD), such as ventricular septal defects and atrial septal defects at birth (9). Using the current projections for heat extremes in the following decades, hundreds of thousands more cases of CHD are expected in some regions of the United States (10).

\section{Air pollution}

Multiple studies have demonstrated that maternal exposure to air pollutants, such as PM, ground-level ozone $\left(\mathrm{O}_{3}\right)$, traffic-related air pollution, and $\mathrm{NO}_{2}$, during the prenatal period is associated with a myriad of adverse pregnancy outcomes. Fetal exposure to air pollution has been associated with prematurity; LBW; atopic diseases, including asthma, aero allergies, and eczema (11); impaired lung development; neurodevelopmental conditions, such as ASD $(12,13)$; thinner brain cortex and impaired inhibitory control; cognitive impairment; and malignancy. Unabated, the continued use of fossil fuels, along with the resulting temperature increases, heat waves, droughts, and wildfires, are expected to increase the concentration of $\mathrm{O}_{3}, \mathrm{PM}$, and other pollutants; worsen air quality; and increase the adverse health consequences children may have because of intrauterine pollution exposure.
Infections/vector-borne disease Environmental changes suitable for the transmission of some mosquito-borne diseases, such as dengue fever and malaria, continue to increase with climate change (14). At least 125 million women at risk for malaria are estimated to become pregnant every year. Maternal infection with malaria and dengue fever has been associated with small-for-gestational-age babies, prematurity, and LBW $(15,16)$. Likewise, the WHO estimates that $5 \%-15 \%$ of infants born to women infected with the Zika virus during pregnancy have infection-related complications (17).

\section{Conclusion}

It is impossible to predict the scope and impact of climate change in future generations of children. However, the convergence of multiple adverse health outcomes, coming from different pathways of exposure in the prenatal and postnatal life, will likely have a compounding effect that will accelerate or worsen the morbidity and mortality of many health conditions. Long-term outcomes will likely be worsened by minority status, poverty, and socioeconomic disadvantages.

Children are the least responsible for climate change but will bear the greatest burden of its impact. The WHO estimates that close to $90 \%$ of the burden of disease attributable to climate change is borne by children under the age of 5 , in both developing and developed countries (18). It will be difficult to overcome these challenges because almost $20 \%$ of the world's children live in extreme poverty, which further compromises their social status and access to care. As we allocate our public health resources and develop strategies to advance children's health, we need to reflect on where to concentrate our efforts. Although the negative effects of climate change on health may be reduced by improved access to care, improved disaster management, and poverty alleviation, the systemic disregard for children's rights and well-being, which are sacrificed for economic development, is a child's biggest threat. At the personal level, we must reflect on what to do with the knowledge of what the climate crisis is doing and will continue to do to children around the world. We can jump to action or stand in complacent indifference while children continue to die. 
Address correspondence to: Susan E. Pacheco, The University of Texas McGovern Medical School, 6431 Fannin Street, Houston, Texas 77030, USA. Phone: 713.500.5650; Email: susan.e.pacheco@ uth.tmc.edu.

1. Smith KR, et al. 2014: Human health: impacts, adaptation, and co-benefits. In: Field CB, et al., eds. Climate Change 2014: Impacts, Adaptation, and Vulnerability. Part A: Global and Sectoral Aspects. Cambridge, United Kingdom, and New York, New York, USA: Cambridge University Press; 2015:709-754.

2. Ahdoot S, Pacheco SE, Council on Environmental Health. Global climate change and children's health. Pediatrics. 2015;136(5):e1468-e1484.

3. United Nations Children's Fund (UNICEF). The Challenges of Climate Change: Children on the Front Line. Florence, Italy: UNICEF; 2014.

4. Calderón-Garcidueñas L, Kulesza RJ, Doty RL, D’Angiulli A, Torres-Jardón R. Megacities air pollution problems: Mexico City Metropolitan Area critical issues on the central nervous system pediatric impact. Environ Res. 2015;137:157-169.

5. Liu GT, Dancause KN, Elgbeili G, Laplante DP,
King S. Disaster-related prenatal maternal stress explains increasing amounts of variance in body composition through childhood and adolescence: Project Ice Storm. Environ Res. 2016;150:1-7.

6. Dancause KN, et al. Prenatal stress due to a natural disaster predicts adiposity in childhood: the Iowa Flood Study. JObes. 2015;2015:570541.

7. Roseboom TJ, Painter RC, van Abeelen AF, Veenendaal MV, de Rooij SR. Hungry in the womb: what are the consequences? Lessons from the Dutch famine. Maturitas. 2011;70(2):141-145.

8. Mallett LH, Etzel RA. Flooding: what is the impact on pregnancy and child health? Disasters. 2018;42(3):432-458.

9. Lin S, et al. Maternal ambient heat exposure during early pregnancy in summer and spring and congenital heart defects - a large US population-based, case-control study. Environ Int. 2018;118:211-221.

10. Zhang W, et al. Projected changes in maternal heat exposure during early pregnancy and the associated congenital heart defect burden in the United States. J Am Heart Assoc. 2019;8(3):e010995.

11. Deng Q, Lu C, Li Y, Sundell J, Norbäck D. Exposure to outdoor air pollution during trimesters of preg- nancy and childhood asthma, allergic rhinitis, and eczema. Environ Res. 2016;150:119-127.

12. Shukla A, et al. Air pollution associated epigenetic modifications: transgenerational inheritance and underlying molecular mechanisms. Sci Total Environ. 2019;656:760-777.

13. Pagalan L, et al. Association of prenatal exposure to air pollution with autism spectrum disorder. JAMA Pediatr. 2019;173(1):86-92.

14. Watts N, et al. The 2019 report of The Lancet Countdown on health and climate change: ensuring that the health of a child born today is not defined by a changing climate. Lancet. 2019;394(10211):1836-1878.

15. Rijken MJ, et al. Quantifying low birth weight, preterm birth and small-for-gestational-age effects of malaria in pregnancy: a population cohort study. PLOS ONE. 2014;9(7):e100247.

16. Paixão ES, et al. Dengue during pregnancy and live birth outcomes: a cohort of linked data from Brazil. BMJ Open. 2019;9(7):e023529.

17. WHO. Zika virus. http://www.who.int/newsroom/fact-sheets/detail/zika-virus. Updated July 20, 2018. Accessed November 1, 2019.

18. Zhang Y, Bi P, Hiller JE. Climate change and disability-adjusted life years. JEnviron Health. 2007;70(3):32-36. 